\title{
The Phenomenology of Error Processing: The Dorsal ACC Response to Stop-signal Errors Tracks Reports of Negative Affect
}

\author{
Robert P. Spunt ${ }^{1}$, Matthew D. Lieberman ${ }^{1}$, Jessica R. Cohen ${ }^{1,2}$, \\ and Naomi I. Eisenberger ${ }^{1}$
}

\begin{abstract}
A reliable observation in neuroimaging studies of cognitive control is the response of dorsal ACC (dACC) to events that demand increased cognitive control (e.g., response conflicts and performance errors). This observation is apparently at odds with a comparably reliable association of the dACC with the subjective experience of negative affective states such as pain, fear, and anxiety. Whereas "affective" associates of the dACC are based on studies that explicitly manipulate and/or measure the subjective experience of negative affect, the "cognitive" associates of dACC are based on studies using tasks designed to manipulate the demand for cognitive control, such as the Stroop, flanker, and stop-signal tasks. Critically, extant neuroimaging research has not systematically considered the extent to which these cognitive tasks induce negative affective experiences and, if
\end{abstract}

\section{INTRODUCTION}

By the late 1990s, one of the most reliable observations in cognitive neuroscience was the response of the dorsal region of the ACC (dACC; the region of ACC superior and posterior to the genu of the corpus callosum) to the performance of cognitively engaging tasks, especially to events that demand increases in cognitive control (Duncan \& Owen, 2000; Paus, Koski, \& Caramanos, 1998). Even earlier, electrophysiological investigations had identified an event-related brain potential robustly associated with performance errors assumed to be generated by the dACC (Gehring, Goss, Coles, Meyer, \& Donchin, 1993; Falkenstein, Hohnsbein, Hoormann, \& Blanke, 1991). The reliability of these observations paved the way for a still predominant account of the dACC as an informationprocessing hub that operates to detect and/or signal the need for adjustments in cognitive control (Ridderinkhof, Ullsperger, Crone, \& Nieuwenhuis, 2004; Yeung, Botvinick, \& Cohen, 2004; Holroyd \& Coles, 2002; Botvinick, Braver, Barch, Carter, \& Cohen, 2001), and early on, this "cognitive" account of dACC was categorically distinguished

${ }^{1}$ University of California, Los Angeles, ${ }^{2}$ University of California, Berkeley so, to what extent negative affect can account for any variance in the dACC response during task performance. While undergoing fMRI, participants in this study performed a stop-signal task while regularly reporting their experience of performance on several dimensions. We observed that within-subject variability in the dACC response to stop-signal errors tracked changes in subjective frustration throughout task performance. This association remained when controlling for within-subject variability in subjective reports of cognitive engagement and several performance-related variables indexing task difficulty. These results fit with existing models characterizing the dACC as a hub for monitoring ongoing behavior and motivating adjustments when necessary and further emphasize that such a function may be linked to the subjective experience of negative affect.

from an "emotional" characterization of the rostral subdivision of ACC (rACC; the region of ACC anterior and inferior to the genu of the corpus callosum; Bush, Luu, \& Posner, 2000; Devinsky, Morrell, \& Vogt, 1995).

Although the evidence for an information-processing account of dACC is compelling, there is arguably an equally compelling body of evidence associating the dACC with the subjective experience of negative affective states such as pain, fear, and anxiety (for reviews, see Etkin, Egner, \& Kalisch, 2011; Shackman et al., 2011; Vogt, 2005; Eisenberger \& Lieberman, 2004). As early as the 1950s, cingulotomies-a surgical procedure that lesions a portion of the dACC but not the rACC-were shown to be successful in treating medically intractable pain (e.g., Foltz \& White, 1962) and anxiety disorders (e.g., Whitty, Duffield, \& Tow, 1952). Following cingulotomy for pain, patients reported that they could still detect the presence of pain but that it was no longer distressing (Foltz \& White, 1962), and this observation accords with more recent neuroimaging evidence linking the distress or "felt unpleasantness" of physical pain to the dACC (Rainville, Duncan, Price, Carrier, \& Bushnell, 1997). In addition, the dACC has been consistently linked to feelings of distress following social rejection (e.g., Eisenberger, in press; Eisenberger, Lieberman, \& Williams, 2003) and negative 
affect inductions designed to produce fear, anger, or disgust (Etkin et al., 2011; Shackman et al., 2011; Kober et al., 2008).

Taken together, the evidence is compelling that the dACC is linked to the subjective experience of negative affective states. Yet tasks specifically designed to manipulate cognitive control demands, such as the Stroop, flanker, and stop-signal, reliably produce activation in the dACC, especially on trials featuring performance errors. Critically, it is unknown to what extent these tasks induce experiences of negative affect, and if so, whether these experiences explain any part of the dACC response to the task. In other words, we still have not answered the question posed by Rainville (2002): "How does it feel when ACC is activated during a cognitive task?" (p. 198).

There is indirect evidence to suggest that dACC activation during cognitive performance, in particular to performance errors, may be associated with negative affective experiences. The majority of this evidence comes from work examining factors that influence the magnitude of the error-related negativity (ERN; for a recent review, see Weinberg, Riesel, \& Hajcak, 2012), an event-related brain potential reliably associated with error commission and source-localized to the dACC (Debener et al., 2005). Early on, Luu, Collins, and Tucker (2000) showed that during performance of a flanker task, the magnitude of the ERN was associated with individual differences in state and trait negative affect. Other work has shown that the ERN is associated with increased autonomic (Hajcak, McDonald, \& Simons, 2003) and startle (Hajcak \& Foti, 2008) response to errors. Moreover, individuals diagnosed with psychopathologies where negative affect is a core feature exhibit an enhanced dACC response to performance errors (Weinberg, Olvet \& Hajcak, 2010; Olvet, \& Hajcak, 2008; Chiu \& Deldin, 2007; Fitzgerald et al., 2005; Gehring, Himle, \& Nisenson, 2000). In addition to studies demonstrating a link between the ERN and negative affect, studies using fMRI have observed that that the amplitude of the dACC response during cognitive performance is associated with variation in autonomic arousal (Critchley, Tang, Glaser, Butterworth, \& Dolan, 2005) and the self-reported desire to avoid a task (McGuire \& Botvinick, 2010). On the basis of findings such as these, Botvinick (2007) proposed that the types of events that reliably activate the dACCnamely, response conflicts and performance errors-may be intrinsically aversive and that their aversiveness may be pivotal to motivating adjustments in cognitive control.

The studies just reviewed demonstrate a link between numerous correlates of negative affective experiences (e.g., skin conductance, startle response, anxiety disorder) and the dACC response to cognitive performance. However, it remains unknown to what extent dACC activation during cognitive performance is associated with the subjective experience of negative affect in response to such performance. This study was designed to answer this question. To do so, we had participants undergo fMRI while performing a standard version of the stop-signal task (SST; Logan, 1994), which is commonly used to investigate cognitive control and which reliably activates the dACC (e.g., Cohen et al., 2010). In addition, we assessed natural variability in the subjective experience of performing the task to test the hypothesis that within-subject variability in the dACC response to task performance would be associated with within-subject variability in self-reported negative affect.

\section{METHODS \\ Participants}

Twenty-one participants were recruited from either the University of California, Los Angeles (UCLA) subject pool or the surrounding community, provided informed consent according to the procedures of the UCLA Institutional Review Board, and received monetary compensation for participating. Participants were screened to be safe for the MR environment, not diagnosed with a mental illness, and not taking psychotropic medications. Six participants were excluded from the final image analysis because of technical issues (1; head coil malfunction), abnormal brain (1; cavernous malformation in the right frontal lobe), poor SST performance (2; defined a priori as meeting at least one of the following criteria: response rate on the Go task of less than 90\%; more than 10\% incorrect trials on the Go task; percentage of inhibition on the Stop task less than $25 \%$ or greater than $75 \%$; or a stop-signal RT [SSRT] of less than $80 \mathrm{msec}$; Cohen et al., 2010), or a lack of an emotional response to the task (2; defined a priori as not meeting the criterion, described further below, for classifying a participant as demonstrating an emotional response to the SST). These exclusion procedures left 15 participants (nine women, mean age $=21.87$ years, age range $=19-28$ years) for the analyses.

\section{Experimental Design and Procedure}

Participants performed four runs of the SST (Logan, 1994), which features frequent "Go" trials, which require a speeded response, and less frequent "Stop" trials, which require the response to be withheld (Figure 1A). The Go task was a two-choice RT task with spatially compatible stimulus-response mappings: participants used their right hand to press a left button with their index finger if an arrow pointed leftward and a right button with their middle finger if an arrow pointed rightward. Before each trial, a warning signal (open circle) appeared on the monitor for $500 \mathrm{msec}$ (visual angle subtended $4.9^{\circ} \times 4.9^{\circ}$ ). An arrow then appeared in the center of the circle until either the participant responded or 1000 msec elapsed.

On $25 \%$ of trials, a tone was sounded at a variable delay (the stop-signal delay [SSD]) after the onset of the arrow stimulus; this served as a signal for participants to inhibit 
Figure 1. The SST. (A) Schematic of go and stop trials. (B) Schematic of Go and Stopsignal blocks. (C) Mean selfreports of each dimension of negative affect for Stop-signal and Go blocks in the behavioral pilot study $(n=39)$.
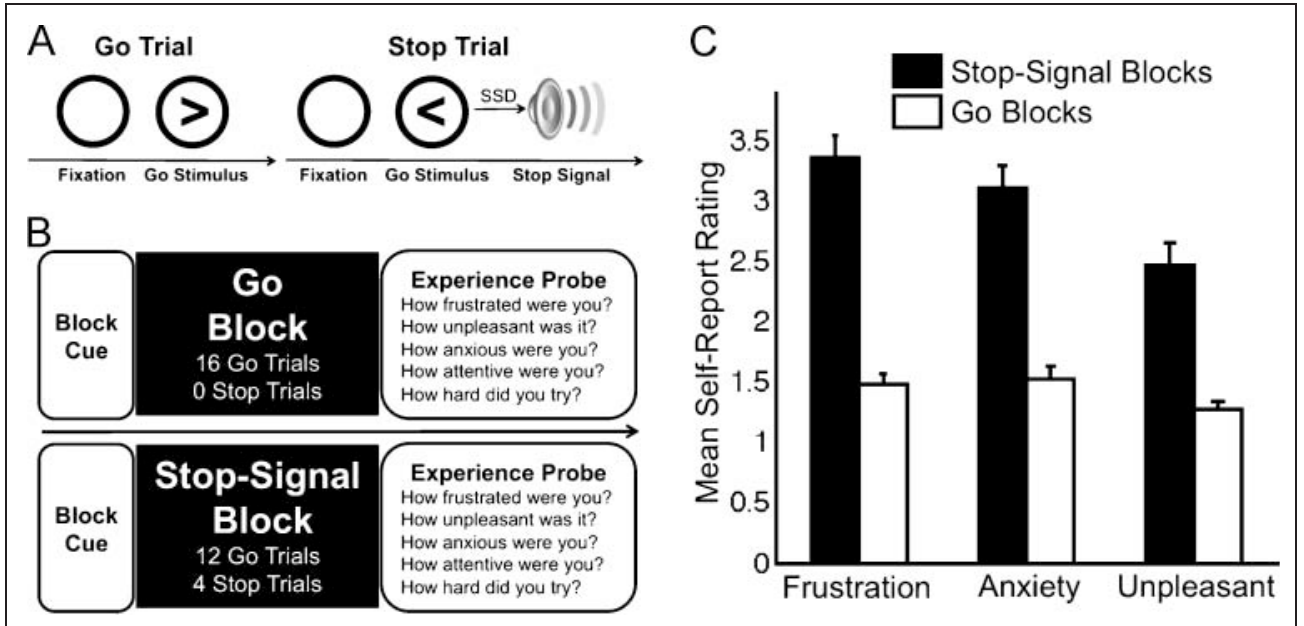

their manual response. If participants failed to inhibit, the trial proceeded as if it were a Go trial. If participants successfully inhibited, the arrow remained on the screen for 1000 msec. Error rates were controlled using an adaptive tracking procedure (1 up, 1 down staircase) wherein the SSD was adjusted throughout performance of the task to produce approximately 50\% successful inhibition for each participant. Two independent staircase functions with a step size of $50 \mathrm{msec}$ were used, with starting delays on the first run of 250 and $350 \mathrm{msec}$. For Runs 2-4, the final SSD of each staircase on the previous run was used as the starting values.

The first run was administered immediately before functional scanning during an anatomical scan; the purpose of this run was to ensure participants understood the task. This run contained 128 trials (96 Go, 32 Stop) performed without interruption. For every eight trials, there were four left and four right arrows and two stop trials (one per ladder). Order of arrow direction and Stop trial ladder were randomized. The position of the stop-signals was pseudorandomized with the constraint that the stop-signal could not occur on three or more contiguous trials. A blank screen was presented for a fixed interval of $500 \mathrm{msec}$ between trials (ISI).

The remaining three runs were during fMRI acquisition (Figure 1B). These runs were identical to the first run, except for the following changes: (a) each run was divided into eight blocks of 16 trials, and for two of these blocks, stop-signals were removed; (b) before each block, participants were presented with a screen informing them whether the upcoming block contained stopsignals; (c) after each block, participants completed an experience probe (described below); and (d) the ISI was jittered between 500 and 4000 msec (mean = 1000 msec, sampled from an exponential distribution).

At each probe, participants reported their experience of the preceding block on three dimensions of negative affect: frustration (How frustrated were you?), anxi- ety (How anxious were you?), and felt unpleasantness (How unpleasant was it?). In a pilot study of 39 UCLA undergraduates, we found that, compared with go-only blocks, stop-signal blocks significantly increased reports of negative affect on these three dimensions (Figure $1 \mathrm{C}$ ). In addition to these dimensions of negative affect, we also assessed two dimensions of cognitive engagement at each probe: attention (How attentive were you?) and effort (How hard did you try?). Participants responded using a 7-point Likert scale $(1=$ not at all, $3=$ slightly, $5=$ quite $a$ bit, $7=$ extremely). To use the scale, participants used three buttons with their index, middle, and ring fingers: The first button decreased the response value, the second button increased the response value, and the third button committed the response value. The starting value was always anchored at 1 . Participants were given $20 \mathrm{sec}$ to complete each probe.

The MATLAB (The MathWorks, Inc., Natick, MA) Psychophysics Toolbox (Brainard, 1997) version 7.4 was used to present the stimuli to participants and to record their responses. Participants viewed the task through LCD goggles and responded using an MR-compatible button box.

\section{Image Acquisition}

Imaging data were acquired using a Siemens Trio 3.0-T MRI scanner at the UCLA Ahmanson-Lovelace Brainmapping Center. For each participant, we acquired 828 functional T2*-weighted EPI volumes (slice thickness $=4 \mathrm{~mm}$, 34 slices, repetition time $[\mathrm{TR}]=2000 \mathrm{msec}$, echo time $[\mathrm{TE}]=30 \mathrm{msec}$, flip angle $=90^{\circ}$, matrix $=64 \times 64$, field of view $=192 \mathrm{~mm}$ ) divided evenly across three runs. We also acquired a T2-weighted matched-bandwidth anatomical scan (same parameters as EPIs, except for $\mathrm{TR}=5000 \mathrm{msec}, \mathrm{TE}=34 \mathrm{msec}$, flip angle $=90^{\circ}$, matrix $=$ $128 \times 128$ ) and a T1-weighted magnetization-prepared rapid acquisition gradient-echo anatomical scan (slice 
thickness $=1 \mathrm{~mm}, 176$ slices, $\mathrm{TR}=2530 \mathrm{msec}, \mathrm{TE}=$ 3.31 msec, flip angle $=7^{\circ}$, matrix $=256 \times 256$, field of view $=256 \mathrm{~mm}$ ).

\section{Performance and Experience Analysis}

MATLAB was used to analyze performance and experience data. For SST performance, we computed Go percent accuracy and RT, standard deviation of Go RT, percentage of responding on Go trials, percentage of successful inhibition, and SSRT. Only correct Go trials were included in the Go RT calculation. SSRT was calculated according to the race model of stopping (Logan \& Cowan, 1984). The race model assumes that the Go and Stop processes occur in parallel and are stochastically independent, although when using the tracking method, this assumption is not critical (Band, Van der Molen, \& Logan, 2003). To calculate SSRT, we first arranged all correct Go RTs in an assumption-free distribution in ascending order. Next, we determined the proportion of failed inhibition. The RT corresponding to that proportion was computed: the quantile RT (i.e., if failed inhibition was .55 , the RT corresponding to $55 \%$ of the area under the RT distribution curve was the quantile RT). SSRT was calculated as the difference between the quantile RT and the average SSD.

For SST experience, we computed mean ratings for the five experience dimensions for both Stop-signal and Go blocks (Figure 1B) and used paired-sample $t$ tests to test differences across the two block types. For Stop-signal blocks, we computed within-subject correlations among time courses for the five measured experience dimensions, the number of failed inhibitions per block, and the average SSD per block.

We used a liberal criterion for classifying participants as demonstrating an emotional response to the SST. Namely, participants were considered responders only when their mean emotion rating was greater than 1.25 during the Stop-signal blocks (scale range, 1-7). Using this criterion, 15 participants were classified as demonstrating a frustration response $\left(\right.$ mean $_{\text {resp }}=2.42$, mean $\left._{\text {non-resp }}=1.19\right)$, 13 as demonstrating an anxious response (mean $_{\text {resp }}=$ 2.17 , mean $_{\text {non-resp }}=1.06$ ), and 11 as demonstrating an unpleasant response $\left(\right.$ mean $_{\text {resp }}=1.91$, mean $\left._{\text {non-resp }}=1.05\right)$. These relatively high exclusion rates on the basis of a lack of an emotional response were not anticipated based on the pilot study. Using this same criterion, 97\% of the participants in the pilot study reported feeling anxiety and frustration, whereas $82 \%$ reported feeling unpleasantness. We note two explanations for the attenuated emotional response to the SST in the imaging sample relative to the pilot sample. The first regards the well-documented fact that undergoing an MRI is itself emotionally distressing (Quirk, Letendre, Ciottone, \& Lingley, 1989). Given this, it is plausible that some participants in the imaging sample may have been at or near ceiling in their baseline levels of negative affect. The second regards a major procedural difference across the pilot and imaging samples. As described above, participants in the imaging sample performed a practice run of the SST in which self-reports were not collected; participants in the pilot sample did not perform this practice run. Hence, it is possible that the emotional response to the task partially habituated during this period.

\section{Image Analysis}

Functional data were analyzed using SPM (SPM8, Wellcome Department of Cognitive Neurology, London, United Kingdom) implemented in MATLAB. Within each run, image volumes were slice time corrected; realigned to correct for head motion; normalized into Montreal Neurological Institute $(\mathrm{MNI})$ space (resampled at $3 \times$ $3 \times 3 \mathrm{~mm}$ ) using the default SPM segmentation routine; smoothed with an 8-mm Gaussian kernel, FWHM; detrended using the linear model of the global signal method (Macey, Macey, Kumar, \& Harper, 2004); and standardized so that the mean of each voxel's time series was 100. Following this preprocessing, image volumes from all runs were concatenated to produce a single consecutive time series. This procedure of detrending and standardizing the time course of each run separately before their concatenation allows us to capitalize on between-run variability in the emotional response in the statistical analysis and follows procedures used in previous work (e.g., Zaki, Weber, Bolger, \& Ochsner, 2009).

For each subject, we began by estimating three models (henceforth referred to as the "Base Models"), one for each of the three dimensions of negative affect (frustration, anxiety, felt unpleasantness). In each of these models, we separately modeled the three events that occur most frequently during performance of the SST: accurate Go responses (Go-success), successful inhibitions for Stop trials (Stop-success), and failed inhibitions for Stop trials (Stop-failures). The onset of each event was defined as the onset of the Go (arrow) stimulus. Regressors were created by convolving a delta function at each event onset with a canonical (double-gamma) hemodynamic response function. In addition to the regressors modeling the average (time-invariant) response, each model also included one of the three dimensions of negative affect as a blockwise parametric modulator of the response to the three events of interest occurring with each block. These parametric regressors were created by modulating the amplitude of the delta functions at each event by the demeaned values of the parameter (e.g., self-reported frustration) for each trial. Within these models, our primary goals were to estimate both the parametric effect of negative affect on each of the three event types, as well as the difference in parametric effects across the event types. These parametric contrasts allowed us to determine regions where the relationship between negative affect and brain activity differs among the three event types.

To further specify the effect of frustration on the dACC response to Stop-failure trials, we estimated one additional model (referred to as the "Control Model"). This model 
was identical to the Base Model for frustration, except it included four additional parametric modulators of the response to Stop-failures. These were (1) trial-wise variability in the latency of the erroneous manual response, (2) trialwise variability in the SSD, (3) block-wise variability in the total number of errors occurring within each block, and (4) block-wise variability in subjective cognitive engagement. The latter parameter was computed by averaging the time courses of self-reported attentiveness and effort. The latter two measures were averaged into a composite because their time courses were highly intercorrelated $(r=.55)$. As in the Base Models, all parameter regressors were created by modulating the amplitude of the delta functions at each event by the parameter's demeaned values.

All models included two additional regressors coding for the position of the three runs in the concatenated time series, and the time series at each voxel was high-pass filtered to $1 / 128 \mathrm{~Hz}$. Serial autocorrelations were modeled as an AR(1) process. For each model, linear contrasts were performed for comparisons of interest, and contrast images were then subjected to one-sample $t$ tests for group analysis. Our primary analyses were conducted within a dACC ROI (Figure 2A). We defined this ROI to capture the putatively cognitive, supracallosal region of the anterior midcingulate (Bush et al., 2000). To produce this ROI, we took the following steps: (a) we created a binary ACC mask using the automated anatomical labeling atlas (Tzourio-Mazoyer et al., 2002), (b) trimmed the mask in the posterior-anterior plane $(0 \leq y \leq 36)$, and (c) overlaid the mask on the average of the participants' normalized anatomical images and edited it to minimize white matter coverage and maximize gray matter coverage.
Analyses within the dACC ROI were performed using a cluster-level family-wise error (FWE) rate of .05, with clusters defined using a voxel-wise threshold of $p<.005$ (uncorrected). We followed all ROI analyses with wholebrain analyses using a cluster-level FWE rate of .05 and a cluster-defining threshold of .001 (uncorrected). For visual presentation, $t$-statistic maps were overlaid on the average of the participants' normalized anatomical images.

\section{RESULTS}

\section{Performance on and Experience of the SST}

Table 1 summarizes all performance and experience data. Performance variables were consistent with past research using the SST (Cohen et al., 2010; Congdon et al., 2010). Importantly, we observed that during Stop-signal blocks, compared with Go blocks, individuals reported experiencing more frustration, $t_{14}=5.87, p<.001\left(M_{\text {diff }}=1.07\right.$, $\left.S D_{\text {diff }}=0.70\right) ;$ anxiety, $t_{14}=2.81, p=.01\left(M_{\text {diff }}=.48\right.$, $\left.S D_{\text {diff }}=.67\right)$; and felt unpleasantness, $t_{14}=4.86, p<$ $.001\left(M_{\text {diff }}=0.49, S D_{\text {diff }}=.39\right)$. This replicates results from the behavioral pilot study (Figure 1C) and confirms that participants subjectively experienced negative emotions in response to performing the SST during the scanning session. Participants also reported being marginally more attentive, $t_{14}=1.95, p=.07\left(M_{\text {diff }}=0.31, S D_{\text {diff }}=.62\right)$, and exerting marginally more effort, $t_{14}=2.08, p=.06$ $\left(M_{\text {diff }}=0.29, S D_{\text {diff }}=.54\right)$, during Stop-signal compared with Go blocks.

Table 2 summarizes all within-subject correlations among the five measured dimensions of experience and relevant

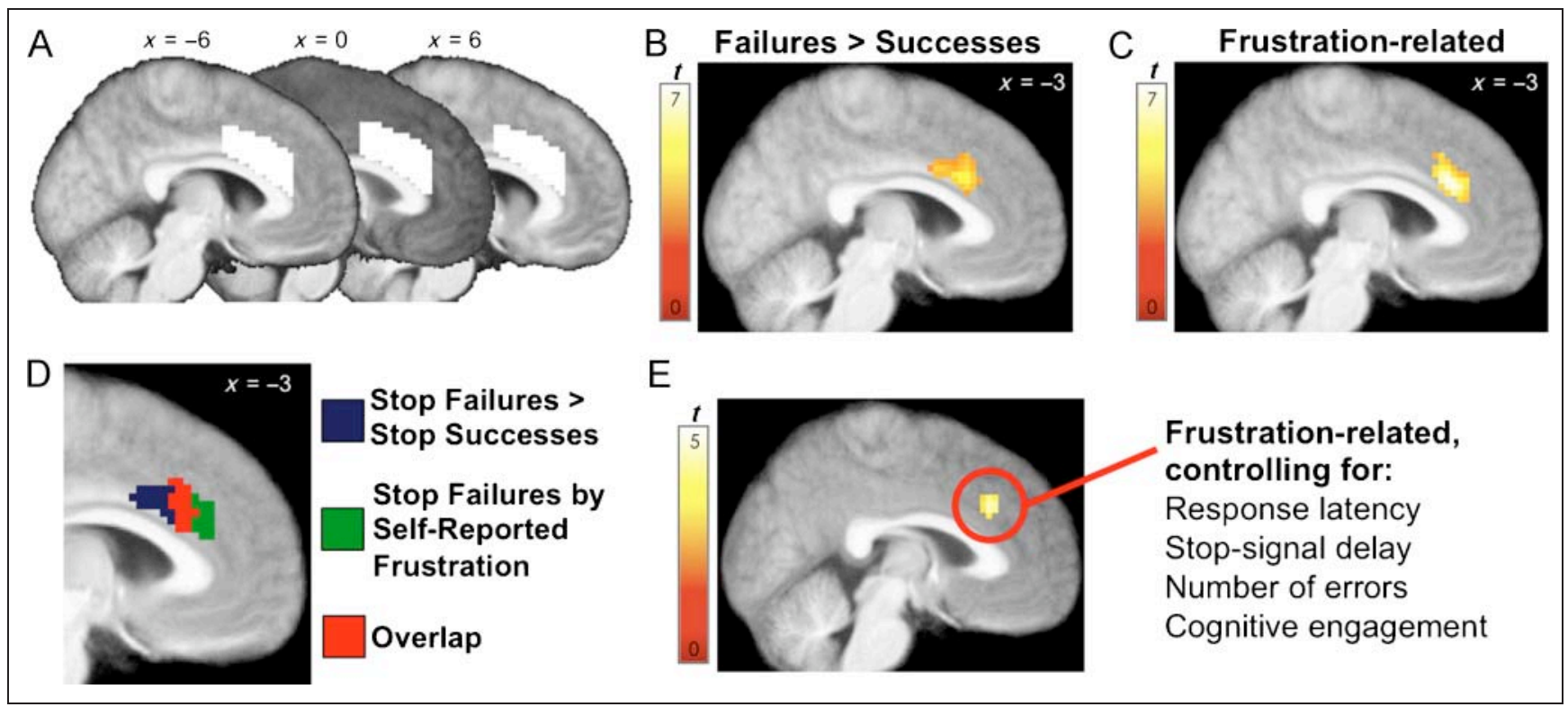

Figure 2. Results observed within dACC ROI analyses. All results are cluster-level corrected using a FWE rate of .05. (A) The dACC mask used in ROI analyses. (B) The region of dACC emerging in the contrast Stop-failures $>$ Stop-successes. (C) The region of dACC where within-subject variability in the magnitude of the response to Stop-failures was associated with within-subject variability in self-reported frustration. (D) Overlap of the regions of dACC observed in B and C. (E) The region of dACC whose response to Stop-failures was associated with self-reported frustration in the Control Model. 
Table 1. Descriptive Statistics of SST Performance and Experience for All Participants Included in the Analysis $(n=15)$

\begin{tabular}{|c|c|c|c|c|}
\hline Variable & Mean & $S D$ & Min & $\operatorname{Max}$ \\
\hline Go accuracy & 99.70 & 0.57 & 97.92 & 100.00 \\
\hline Median Go RT & 581.60 & 109.16 & 370.40 & 746.18 \\
\hline$S D$ Go RT & 133.17 & 28.65 & 91.20 & 175.11 \\
\hline Percentage of Go responses & 98.01 & 2.30 & 91.67 & 100.00 \\
\hline Percentage of inhibition & 53.10 & 6.42 & 41.67 & 64.58 \\
\hline SSRT & 168.87 & 27.20 & 128.00 & 225.30 \\
\hline \multicolumn{5}{|l|}{ Frustration } \\
\hline Stop-signal & 2.42 & 0.71 & 1.50 & 3.89 \\
\hline Go & 1.36 & 0.47 & 1.00 & 2.83 \\
\hline \multicolumn{5}{|l|}{ Anxiety } \\
\hline Stop-signal & 2.02 & 0.85 & 1.00 & 4.00 \\
\hline Go & 1.53 & 0.53 & 1.00 & 2.67 \\
\hline \multicolumn{5}{|l|}{ Unpleasant } \\
\hline Stop-signal & 1.69 & 0.57 & 1.00 & 3.00 \\
\hline Go & 1.20 & 0.39 & 1.00 & 2.50 \\
\hline \multicolumn{5}{|l|}{ Attention } \\
\hline Stop-signal & 5.51 & 0.76 & 4.50 & 6.83 \\
\hline Go & 5.19 & 1.01 & 3.33 & 6.50 \\
\hline \multicolumn{5}{|l|}{ Effort } \\
\hline Stop-signal & 5.59 & 0.57 & 4.56 & 6.46 \\
\hline Go & 5.30 & 0.89 & 3.33 & 6.75 \\
\hline
\end{tabular}

performance variables that demonstrated intrasubject variation. Correlations among the dimensions of experience were moderate to low, with attentiveness and effort showing the strongest relationship. This indicates that these dimensions tap unique aspects of the phenomenology of task performance. The only association observed among experience and performance was between frustration and block-by-block variation in the number of Stop-failures, $r_{\text {mean }}=.33, t_{14}=3.73, p=.002$.

\section{Neural Correlates of the Experience of the SST}

As described in the Methods, we conducted all primary analyses within a dACC ROI (Figure 2A). All whole-brain results all listed in Table 4. Our analysis began with confirming the presence of a dACC response to Stop-failures in the contrast Stop-failures $>$ Stop-successes. This revealed a cluster (Figure 2B; listed in Table 3 ) in a region of the dACC comparable with error-related activity identified in meta-analyses (e.g., Ridderinkhof et al., 2004).

Next, we used parametric modulation analyses to test if within-subject variability in the subjective experience of any of the three dimensions of negative affect were associated with the amplitude of the dACC response to any of three basic events that occur during the task: Gosuccesses, Stop-successes, and Stop-failures. Reports of frustration did not significantly explain any variability in the dACC response to either Go- or Stop-successes. However, frustration did significantly explain variability in the response to Stop-failures, both in the ROI and whole-brain analyses (Figure 2C; listed in Tables 3 and 4). As shown in Figure 2D, this cluster demonstrates considerable overlap with the region of dACC observed in the contrast Stopfailure $>$ Stop-success, although it is notable that there is some clear differentiation, with the average error effect located more caudally than the parametric effect of frustration. To determine how specific this effect was to error trials, we directly contrasted the parametric effect of frustration on Stop-failures to the same effect estimated for both Go-successes and Stop-successes. For both contrasts, we observed a cluster in dACC overlapping with the cluster depicted in Figure 1C (listed in Table 3). This demonstrates that the link between the dACC response and self-reported frustration is selectively driven by the response to error trials.

Table 2. Average Within-subject Time Course Correlations among the Dimensions of Experience, Number of Errors per Block, and Average SSD per Block, for All Participants Included in the Analysis $(n=15)$

\begin{tabular}{|c|c|c|c|c|c|c|c|}
\hline & Frustration & Anxiety & Unpleasant & Attention & Effort & Errors & SSD \\
\hline Frustration & 1 & $0.25 *$ & 0.08 & 0.09 & 0.06 & $0.33 *$ & 0.07 \\
\hline Anxiety & - & 1 & $0.33 *$ & 0.14 & 0.15 & 0.01 & 0.02 \\
\hline Unpleasant & - & - & 1 & $0.22 *$ & 0.10 & 0.13 & -0.09 \\
\hline Attention & - & - & - & 1 & $0.55^{*}$ & 0.02 & -0.15 \\
\hline Effort & - & - & - & - & 1 & -0.08 & -0.13 \\
\hline Errors & - & - & - & - & - & 1 & $0.15 *$ \\
\hline
\end{tabular}

*Significantly different from zero at $p<.05$. 
Next, we examined whether variability in felt unpleasantness and/or anxiety explained variance in the dACC response to the SST. For both affective dimensions and for the three event types, no clusters were observed that survived correction in either the whole-brain or dACC ROI analyses. Given the relatively low number of participants showing evidence of experiencing unpleasantness $(n=11)$ and anxiety $(n=13)$ in response to the task, it is notable that two clusters do emerge when interrogating the dACC ROI using the cluster-defining threshold of $p<$ .005 . Namely, we observed associations between reports of felt unpleasantness and the dACC response to Stop-failures (peak: $x=0, y=24, z=33 ; t_{10}=5.77$, voxel extent $=11$ ) and between reports of anxiety and the dACC response to Go-successful events (during stop-signal blocks; peak: $x=$ $3, y=18, z=24 ; t_{12}=3.85$, voxel extent $=15$ ). Although preliminary, these results suggest that dimensions of negative affect other than frustration may explain variance in the dACC response during performance of the SST. However, given that they did not survive correction for multiple comparisons, we focused the remainder of our analyses on the dimension of frustration.

Our final set of analyses tested the specificity of the observed association among self-reported frustration and the dACC response to errors. To do so, we estimated an additional model that was identical to the model in which the relationship between frustration and the response to Stop-failures was observed, except it included parametric regressors modeling the effect of four additional variables on the response to Stop-failures: (1) trialwise variability in the latency of the erroneous manual response, (2) trial-wise variability in the SSD, (3) block-wise variability in the total number of errors occurring within each block, and (4) block-wise variability in subjective cognitive engagement (average of self-reported attentiveness and effort). Within this model, we continued to observe a robust relationship between the dACC response to Stopfailures and subjective frustration (Figure 2E; listed in Table 3). When examining the four additional parametric effects, only one-response latency_explained significant variance in the response of the dACC to Stop-failures. This effect replicates previous work demonstrating that the dACC is sensitive to response latency (Grinband et al., 2011; McGuire \& Botvinick, 2010).

\section{DISCUSSION}

Although the earliest work on dACC function, involving cingulotomies for pain, anxiety, and depression, all

Table 3. Significant Clusters Identified in the Base and Control Models when Searching within the dACC ROI

\begin{tabular}{|c|c|c|c|c|c|}
\hline Tested Effect & $x$ & $y$ & $z$ & $t$ & $k$ \\
\hline \multicolumn{6}{|l|}{ Contrast of Main Effects in the Base Model } \\
\hline Stop-failures $>$ Stop-successes & -12 & 24 & 30 & 7.00 & 104 \\
\hline \multicolumn{6}{|l|}{ Parametric Effects in the Base Model } \\
\hline Frustration: Stop-failures & -6 & 27 & 27 & 6.28 & 125 \\
\hline Frustration: Stop-successes & \multicolumn{5}{|c|}{ No significant clusters } \\
\hline Frustration: Go-successes & \multicolumn{5}{|c|}{ No significant clusters } \\
\hline \multicolumn{6}{|c|}{ Contrasts of Parametric Effects in the Base Model } \\
\hline Frustration: Stop-failures $>$ Stop-successes & -6 & 21 & 24 & 4.07 & 50 \\
\hline Frustration: Stop-failures > Go-successes & 0 & 21 & 27 & 4.43 & 93 \\
\hline \multicolumn{6}{|c|}{ Parametric Effects (for Stop-failures Only) in the Control Model } \\
\hline Frustration & -6 & 27 & 30 & 4.41 & 24 \\
\hline Response Latency & -9 & 24 & 30 & 5.19 & 119 \\
\hline SSD & \multicolumn{5}{|c|}{ No significant clusters } \\
\hline Number of errors & \multicolumn{5}{|c|}{ No significant clusters } \\
\hline Subjective engagement & \multicolumn{5}{|c|}{ No significant clusters } \\
\hline
\end{tabular}

All clusters were identified using a voxel-wise threshold of $p<.005$ (uncorrected) and are significant at a cluster-level FWE rate of .05 .

$n=15 . x, y$, and $z$ refer to MNI coordinates of local maxima in the left-right, anterior-posterior, and inferior-superior dimensions, respectively. $t$ refers to the $t$ statistic at those coordinates. $k$ refers to the cluster size (in voxels). 
Table 4. Significant Clusters Identified in the Base and Control Models when Searching within the Whole Brain

\begin{tabular}{|c|c|c|c|c|c|c|}
\hline Anatomical Region & & $x$ & $y$ & $z$ & $t$ & $k$ \\
\hline \multicolumn{7}{|c|}{ Stop-failures > Stop-successes (Base Model) } \\
\hline dACC & $\mathrm{L}$ & -12 & 24 & 30 & 7.00 & 43 \\
\hline Pregenual cingulate cortex & $\mathrm{L}$ & -12 & 48 & 6 & 5.93 & 76 \\
\hline Dorsomedial pFC & $\mathrm{L}$ & -15 & 51 & 27 & 4.77 & - \\
\hline Cerebellum & $\mathrm{R}$ & 15 & -57 & -18 & 5.43 & 92 \\
\hline Pre-SMA & $\mathrm{L} / \mathrm{R}$ & -3 & 15 & 57 & 5.41 & 59 \\
\hline Midbrain & $\mathrm{L} / \mathrm{R}$ & 6 & -24 & -12 & 5.09 & 45 \\
\hline \multicolumn{7}{|c|}{ Stop-successes > Stop-failures (Base Model) } \\
\hline Middle occipital gyrus & $\mathrm{L}$ & -33 & -90 & 15 & 7.42 & 346 \\
\hline Middle occipital gyrus & $\mathrm{R}$ & 42 & -72 & -12 & 7.46 & 513 \\
\hline Putamen & $\mathrm{R}$ & 27 & 12 & -6 & 6.27 & 45 \\
\hline
\end{tabular}

Parametric Effect of Frustration on Stop-failures (Base Model)

Positive effect

dACC

L $\quad-6$

Negative effect

Postcentral gyrus

SMA

$\mathrm{L}$

$-30$

$-36$

57

5.32

210

$\mathrm{L} / \mathrm{R}$

3

$-15$

57

Parametric Effect of Frustration on Stop-successes (Base Model)

No significant clusters

Parametric Effect of Frustration on Go-successes (Base Model)

No significant clusters

Contrasts of Parametric Effects of Frustration (Base Model)

Stop-failures $>$ Go-successes

dACC

Go-successes > Stop-failures

Postcentral gyrus

Stop-failures $>$ Stop-successes

No significant clusters

Stop-successes $>$ Stop-failures

Postcentral gyrus

L

L

L

$-6$

30

$-33$

57

5.69

124

Go-successes > Stop-successes

No significant clusters

Stop-successes > Go-successes

No significant clusters

3 76 (1) 45 46 13

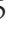


Table 4. (continued)

\begin{tabular}{|c|c|c|c|c|c|c|}
\hline Anatomical Region & & $x$ & $y$ & $z$ & $t$ & $k$ \\
\hline \multicolumn{7}{|c|}{ Parametric Effects on Stop-failures Estimated in the Control Model } \\
\hline \multicolumn{7}{|l|}{ Frustration } \\
\hline \multicolumn{7}{|l|}{ Positive effect } \\
\hline \multicolumn{7}{|l|}{ No significant clusters } \\
\hline \multicolumn{7}{|l|}{ Negative effect } \\
\hline Postcentral gyrus & $\mathrm{L}$ & -33 & -36 & 57 & 6.17 & 52 \\
\hline \multicolumn{7}{|l|}{ Response latency } \\
\hline \multicolumn{7}{|l|}{ Positive effect } \\
\hline \multirow[t]{2}{*}{ Anterior insula/inferior frontal gyrus } & $\mathrm{R}$ & 33 & 24 & 6 & 6.17 & 170 \\
\hline & $\mathrm{L}$ & -36 & 30 & -3 & 5.56 & 97 \\
\hline SMA & $\mathrm{R}$ & 12 & 9 & 66 & 5.96 & 33 \\
\hline Superior temporal gyrus & $\mathrm{L}$ & -45 & -9 & -9 & 5.14 & 38 \\
\hline Middle frontal gyrus & $\mathrm{L}$ & 39 & 45 & 9 & 5.13 & 36 \\
\hline \multicolumn{7}{|l|}{ Negative effect } \\
\hline \multicolumn{7}{|l|}{ No significant clusters } \\
\hline \multicolumn{7}{|l|}{ SSD } \\
\hline \multicolumn{7}{|l|}{ No significant clusters } \\
\hline \multicolumn{7}{|l|}{ Number of errors } \\
\hline \multicolumn{7}{|l|}{ No significant clusters } \\
\hline \multicolumn{7}{|l|}{ Subjective engagement } \\
\hline No significant clusters & & & & & & \\
\hline
\end{tabular}

All clusters were identified using a voxel-wise threshold of $p<.001$ (uncorrected) and are significant at a cluster-level FWE rate of .05 .

$n=15$. L and R refer to left and right hemispheres. $x, y$, and $z$ refer to MNI coordinates in the left-right, anterior-posterior, and inferior-superior dimensions, respectively. $t$ refers to the $t$ score at those coordinates (local maxima). $k$ refers to the cluster size (in voxels).

pointed to its role in the experience of negative affect, contemporary theoretical accounts of the dACC tend to focus exclusively on its role in cognitive control. The reliable association of the dACC with the exertion of cognitive control is primarily based on the observation of dACC activity during performance of tasks specifically designed to manipulate cognitive control demands. We have shown that one such commonly used task, the SST, although not designed to investigate negative affect, nevertheless induces experiences of negative affect which vary throughout task performance. Importantly, this natural variability in the experience of negative affectnamely, self-reports of frustration-explained a significant amount of variability in the dACC response during task performance. This strongly suggests that the association of dACC with the experience of negative affect cannot be easily reduced to a mere "cognitive" account of its function. Even in its response to a canonical "cognitive" event - a simple performance error on a speeded response inhibition task - the dACC appears to support an affective function.

\section{Relationship to Previous Studies}

Although the cognitive account of dACC has been dominant for more than 15 years, several reviews and metaanalyses have highlighted the dACC's association with both cognitive control and the experience of negative affect (Weinberg et al., 2012; Etkin et al., 2011; Shackman et al., 2011; Botvinick, 2007; Eisenberger \& Lieberman, 2004). For instance, Shackman and colleagues (2011) marshaled data from 192 neuroimaging studies of negative affect, physical pain, and cognitive control and found that all three categories reliably report activation in an overlapping region of the dACC. Although strongly suggestive, the interpretation of meta-analytically defined overlap is not unambiguous, as it is well accepted that the same region can perform different functions in different contexts 
(Yarkoni, Poldrack, Nichols, Van Essen, \& Wager, 2011; Poldrack, 2006). For instance, from these meta-analyses, it would be invalid to infer that during the performance of a task designed to investigate variation in cognitive demand, the presence of dACC activity indicates the presence of a subjective experience of negative affect. Here, we provide the first demonstration of an overlap in the regions associated with the objectively defined (error $>$ nonerror) and subjectively defined (self-reported frustration) aspects of cognitive performance. Moreover, we have shown that the association of self-reported frustration with the dACC response to errors is independent of self-reported cognitive engagement and several performance-related variables. To recapitulate Rainville's (2002) query, "How does it feel when ACC is activated during a cognitive task?" (p. 198). The results of this study provide the best evidence yet that, at least in the response to performance errors, ACC activation-in particular, the dorsal region typically associated with signaling the need for adjustments in cognitive control-feels like frustration.

\section{Integrative Perspectives on dACC Function}

These two correlates of dACC activity - the presence/absence of error and the magnitude of subjective frustrationinvite consideration of integrative models of dACC function which can accommodate its association with both cognitive control and negative affect. Indeed, the dACC is well positioned to support highly integrative functions, which cut across traditional boundaries between cognition and emotion, as it is densely interconnected with brain systems involved in affect, executive control, and action (Shackman et al., 2011; Pessoa, 2008; Paus, 2001). Eisenberger and Lieberman (2004) make analogy to an alarm in describing the multiple correlates of dACC activity. Any functional alarm system (e.g., smoke alarm) requires two operations: (1) detecting discrepancies from a desired set point (e.g., too many particles of smoke) and (2) generating a signal (e.g., alarm bell) capable of motivating corrective action. The performance-defined contrast of error and nonerror events may isolate the first operation (i.e., detection), whereas the magnitude of self-reported frustration in response to error events may isolate an aspect of the second operation (i.e., signaling). The idea that the dACC is involved in both the detection of problems in ongoing behavior and the motivation of adjustments in future behavior is implied by other integrative models of dACC function (Weinberg et al., 2012; Shackman et al., 2011; Botvinick, 2007). For instance, Botvinick (2007) proposed that the dACC may underlie a form of avoidance learning in which cognitive demand (e.g., response conflicts and errors) is registered as aversive to motivate adjustments in future behavior. In support of this proposed link between the aversiveness of cognitive demand and the dACC, McGuire and Botvinick (2010; see also Botvinick, Huffstetler, \& McGuire, 2009) observed that variability in self-reported desire to avoid a cognitively demanding task tracked the dACC response to the task, although in contrast to the present study this association was not robust when controlling for block-wise variability in response latency and error commission. In support of the proposed link between the aversiveness of cognitive demand and the adjustment of ensuing behavior, several behavioral studies have demonstrated that negative affect during cognitive performance facilitates the deployment of cognitive control (van Steenbergen, Booij, Band, Hommel, \& Does, 2012; van Steenbergen, Band, \& Hommel, 2009, 2010) and informs future decisions regarding the avoidance of cognitively demanding tasks (Kool, McGuire, Rosen, \& Botvinick, 2010). Hence, negative affective experiences may not simply be an incidental byproduct of performing cognitively demanding tasks; rather, they may be central to the way in which individuals use information about cognitive difficulties to adjust their future behavior.

\section{Limitations}

There are several limitations that merit discussion. The first is that our results do not directly bear on computational perspectives on dACC function. This is because functional (computational) and phenomenological (selfreported phenomenal experience) perspectives on mental processes sit squarely at different levels of analysis. However, it is noteworthy that the relationship between self-reported frustration and the dACC response to the task was specific to error events. This provides neural evidence for the face valid association of error commission with the experience of frustration. More importantly, these findings demonstrate that, in the response of the dACC to errors, not all errors are created equal and that, whatever attribute of an error the dACC computes, this attribute is likely intimately linked with feelings of frustration. This study suggests that research on the computational basis of affective states, such as frustration, will likely be critical for fully understanding the nature of the interface between cognition and affect in the dACC.

The second limitation regards the nature of our measurement of negative affect. Namely, although the resolution of our experience probes was at the level of individual blocks (1 every 16 trials), subjective experience undoubtedly varied substantially at the level of individual trials. Choosing to measure experience at the end of blocks of trials was intended to provide a good balance between the temporal resolution of our measurement of experience and the amount of disruption this measurement would have on task performance. Despite the block-level resolution of self-reports, we observed strong evidence for a trialspecific association between dACC and frustration: This association was observed for error trials only, and the strength of this association was significantly stronger than that observed for the other trial types occurring within each block. This suggests that future research on the neural bases of meta-cognitive experiences, such as frustration, might benefit by directing participants to report 
experience only of error trials. This would enable an increase in temporal resolution of negative affect measurement while at the same time minimizing the number of interruptions to task performance.

A related limitation concerns the absence of any nonsubjective measures of negative affect, such as measures of autonomic activity. Although it is no doubt true that such measures would have invited consideration of additional questions, they were not necessary for answering the question of interest in this study: What is the relationship between the subjective experience of negative affect and dACC activity during cognitive performance? In fact, it is already known from both neuroimaging and electrophysiological work that the dACC response to cognitive performance is associated with various measures of autonomic activity (e.g., Critchley et al., 2005; Hajcak et al., 2003; for a review, see Weinberg, et al., 2012). However, it remained unknown to what extent the dACC is associated with self-reports of negative affect during cognitive performance. Although self-reported subjective experience is only one correlate of negative affect, it may be a particularly important correlate. Indeed, selfreport remains a gold standard method in the literature on and clinical assessment of physical pain. As Coghill, McHaffie, and Yen (2003) wrote in discussing the association of subjective reports of pain and the dACC response, "pain is defined by the first-person experiential perspective and must be diagnosed and treated with significant consideration of the subjective report... [The] subjective report will likely remain the single most reliable index of the magnitude of pain" (p. 8542). In this spirit, we would argue that in the measurement of any affective response, such as the frustration felt in response to performing a difficult task, self-report should be treated as the gold standard method.

A final limitation regards the exclusive focus on the emotion of frustration. As a group, the sample reported very low amounts of anxiety and felt unpleasantness. Still, exploratory analyses suggest that these two dimensions may explain the dACC response during cognitive performance. A fruitful direction for future research will be to more systematically examine distinct dimensions of negative affect as they relate to distinct aspects of cognitive performance, such as the monitoring of information processing for errors (captured by Go trials in Stop-signal blocks) and the detection of errors (captured by Stopfailure trials).

\section{Conclusion}

Nearly 75 years ago, Papez (1937) suggested that the ACC “... is the seat of dynamic vigilance by which environmental experiences are endowed with an emotional consciousness" (p. 737). Intriguingly, Papez foreshadowed the two faces of the dACC presented here: a cognitive module for vigilantly monitoring the current state of the organism and an affective alarm that endows events_-such as simple errors in the performance of a cognitive task-with an emotional consciousness. One particularly influential view (Bush et al., 2000) might suggest that Papez's comment linked dACC with the cognitive module and rACC with the affective module. This study underscores the possibility that the dACC is central to both.

\section{Acknowledgments}

The authors thank Edward Kobayashi and John Mezzanotte for their assistance with data collection; Russ Poldrack and Adam Aron for helpful advice in the early stages of the project; and Matthew Botvinick, Wouter Kool, and several anonymous reviewers for comments on the manuscript. For generous support, the authors also thank the Brain Mapping Medical Research Organization, Brain Mapping Support Foundation, Pierson-Lovelace Foundation, The Ahmanson Foundation, William M. and Linda R. Dietel Philanthropic Fund at the Northern Piedmont Community Foundation, Tamkin Foundation, Jennifer Jones-Simon Foundation, Capital Group Companies Charitable Foundation, Robson Family, and Northstar Fund.

Reprint requests should be sent to Naomi I. Eisenberger, Department of Psychology, 1285 Franz Hall, University of California, Los Angeles, Los Angeles, CA 90095-1563, or via e-mail: neisenbe@ ucla.edu.

\section{REFERENCES}

Band, G. P. H., Van der Molen, M. W., \& Logan, G. D. (2003). Horse-race model simulations of the stop-signal procedure. Acta Psychologica, 112, 105-142.

Botvinick, M., Braver, T., Barch, D., Carter, C., \& Cohen, J. (2001). Conflict monitoring and cognitive control. Psychological Review, 108, 624.

Botvinick, M. M. (2007). Conflict monitoring and decision making: Reconciling two perspectives on anterior cingulate function. Cognitive, Affective, \& Behavioral Neuroscience, 7, 356-366.

Botvinick, M. M., Huffstetler, S., \& McGuire, J. T. (2009). Effort discounting in human nucleus accumbens. Cognitive, Affective, \& Behavioral Neuroscience, 9, 16-27.

Brainard, D. H. (1997). The Psychophysics Toolbox. Spatial Vision, 10, 433-436.

Bush, G., Luu, P., \& Posner, M. (2000). Cognitive and emotional influences in anterior cingulate cortex. Trends in Cognitive Sciences, 4, 215-222.

Chiu, P., \& Deldin, P. (2007). Neural evidence for enhanced error detection in major depressive disorder. American Journal of Psychiatry, 164, 608-616.

Coghill, R. C., McHaffie, J. G., \& Yen, Y.-F. (2003). Neural correlates of interindividual differences in the subjective experience of pain. Proceedings of the National Academy of Sciences, U.S.A., 100, 8538-8542.

Cohen, J., Asarnow, R. F., Sabb, F. W., Bilder, R. M., Bookheimer, S. Y., Knowlton, B. J., et al. (2010). Decoding developmental differences and individual variability in response inhibition through predictive analyses across individuals. Frontiers in Human Neuroscience, 4, 1-12.

Congdon, E., Mumford, J. A., Cohen, J. R., Galvan, A., Aron, A. R., Xue, G., et al. (2010). Engagement of large-scale networks is related to individual differences in inhibitory control. Neuroimage, 53, 653-663.

Critchley, H. D., Tang, J., Glaser, D., Butterworth, B., \& Dolan, R. J. (2005). Anterior cingulate activity during error and autonomic response. Neuroimage, 27, 885-895. 
Debener, S., Ullsperger, M., Siegel, M., Fiehler, K., von Cramon, D. Y., \& Engel, A. K. (2005). Trial-by-trial coupling of concurrent electroencephalogram and functional magnetic resonance imaging identifies the dynamics of performance monitoring. Journal of Neuroscience, 25, 11730-11737.

Devinsky, O., Morrell, M., \& Vogt, B. (1995). Contributions of anterior cingulate cortex to behaviour. Brain, 118, 279-306.

Duncan, J., \& Owen, A. M. (2000). Common regions of the human frontal lobe recruited by diverse cognitive demands. Trends in Neurosciences, 23, 475-483.

Eisenberger, N. I. (in press). The pain of social disconnection: Examining the shared neural underpinnings of physical and social pain. Nature Reviews Neuroscience.

Eisenberger, N. I., \& Lieberman, M. D. (2004). Why rejection hurts: A common neural alarm system for physical and social pain. Trends in Cognitive Sciences, 8, 294-300.

Eisenberger, N. I., Lieberman, M. D., \& Williams, K. (2003). Does rejection hurt? An fMRI study of social exclusion. Science (New York, NY), 302, 290-292.

Etkin, A., Egner, T., \& Kalisch, R. (2011). Emotional processing in anterior cingulate and medial prefrontal cortex. Trends in Cognitive Sciences, 15, 85-93.

Falkenstein, M., Hohnsbein, J., Hoormann, J., \& Blanke, L. (1991). Effects of crossmodal divided attention on late ERP components. II. Error processing in choice reaction tasks. Electroencephalography and Clinical Neurophysiology, 78, 447-455.

Fitzgerald, K. D., Welsh, R. C., Gehring, W. J., Abelson, J. L., Himle, J. A., Liberzon, I., et al. (2005). Error-related hyperactivity of the anterior cingulate cortex in obsessive-compulsive disorder. Biological Psychiatry, 57, 287-294.

Foltz, E., \& White, L. (1962). Pain "relief" by frontal cingulumotomy. Journal of Neurosurgery, 19, 89-100.

Gehring, W., Goss, B., Coles, M., Meyer, D., \& Donchin, E. (1993). A neural system for error detection and compensation. Psychological Science, 4, 385-390.

Gehring, W., Himle, J., \& Nisenson, L. (2000). Action-monitoring dysfunction in obsessive-compulsive disorder. Psychological Science, 11, 1-7.

Grinband, J., Savitskaya, J., Wager, T. D., Teichert, T., Ferrera, V. P., \& Hirsch, J. (2011). The dorsal medial frontal cortex is sensitive to time on task, not response conflict or error likelihood. Neuroimage, 57, 303-311.

Hajcak, G., \& Foti, D. (2008). Errors are aversive: Defensive motivation and the error-related negativity. Psychological Science, 19, 103-108.

Hajcak, G., McDonald, N., \& Simons, R. F. (2003). To err is autonomic: Error-related brain potentials, ANS activity, and post-error compensatory behavior. Psychophysiology, 40, 895-903.

Holroyd, C. B., \& Coles, M. G. (2002). The neural basis of human error processing: Reinforcement learning, dopamine, and the error-related negativity. Psychological Review, 109, 679-709.

Kober, H., Barrett, L. F., Joseph, J., Bliss-Moreau, E., Lindquist, K., \& Wager, T. D. (2008). Functional grouping and corticalsubcortical interactions in emotion: A meta-analysis of neuroimaging studies. Neuroimage, 42, 998-1031.

Kool, W., McGuire, J. T., Rosen, Z. B., \& Botvinick, M. M. (2010). Decision making and the avoidance of cognitive demand. Journal of Experimental Psychology: General, 139, 665-682.

Logan, G. (1994). On the ability to inhibit thought and action: A users' guide to the stop signal paradigm. In D. Dagenbach \& T. Carr (Eds.), Inbibitory processes in attention, memory, and language (pp. 189-240). San Diego, CA: Academic Press.
Logan, G., \& Cowan, W. (1984). On the ability to inhibit simple and choice reaction time responses: A model and a method. Journal of Experimental Psychology, 10, 276-291.

Luu, P., Collins, P., \& Tucker, D. (2000). Mood, personality, and self-monitoring: Negative affect and emotionality in relation to frontal lobe mechanisms of error monitoring. Journal of Experimental Psychology: General, 129, 43-60.

Macey, P., Macey, K., Kumar, R., \& Harper, R. (2004). A method for removal of global effects from fMRI time series. Neuroimage, 22, 360-366.

McGuire, J. T., \& Botvinick, M. M. (2010). Prefrontal cortex, cognitive control, and the registration of decision costs. Proceedings of the National Academy of Sciences, U.S.A., 107, 7922-7926.

Olvet, D., \& Hajcak, G. (2008). The error-related negativity (ERN) and psychopathology: Toward an endophenotype. Clinical Psychology Review, 28, 1343-1354.

Papez, J. (1937). A proposed mechanism of emotion. Archives of Neurology \& Psychiatry, 38, 725.

Paus, T. (2001). Primate anterior cingulate cortex: Where motor control, drive and cognition interface. Nature Reviews Neuroscience, 2, 417-424.

Paus, T., Koski, L., \& Caramanos, Z. (1998). Regional differences in the effects of task difficulty and motor output on blood flow response in the human anterior cingulate cortex: A review of 107 PET activation studies. NeuroReport, 9, 37-47.

Pessoa, L. (2008). On the relationship between emotion and cognition. Nature Reviews Neuroscience, 9, 148-158.

Poldrack, R. (2006). Can cognitive processes be inferred from neuroimaging data? Trends in Cognitive Sciences, 10, 59-63.

Quirk, M. E., Letendre, A. J., Ciottone, R. A., \& Lingley, J. F. (1989). Anxiety in patients undergoing MR imaging. Radiology, 170, 463-466.

Rainville, P. (2002). Brain mechanisms of pain affect and pain modulation. Current Opinion in Neurobiology, 12, 195-204.

Rainville, P., Duncan, G. H., Price, D. D., Carrier, B., \& Bushnell, M. C. (1997). Pain affect encoded in human anterior cingulate but not somatosensory cortex. Science (New York, NY), 277, 968-971.

Ridderinkhof, K. R., Ullsperger, M., Crone, E. A., \& Nieuwenhuis, S. (2004). The role of the medial frontal cortex in cognitive control. Science (New York, NY), 306, 443-447.

Shackman, A. J., Salomons, T. V., Slagter, H. A., Fox, A. S., Winter, J. J., \& Davidson, R. J. (2011). The integration of negative affect, pain and cognitive control in the cingulate cortex. Nature Reviews Neuroscience, 12, $154-167$

Tzourio-Mazoyer, N., Landeau, B., Papathanassiou, D., Crivello, F., Etard, O., Delcroix, N., et al. (2002). Automated anatomical labeling of activations in SPM using a macroscopic anatomical parcellation of the MNI MRI single-subject brain. Neuroimage, 15, 273-289.

van Steenbergen, H., Band, G. P. H., \& Hommel, B. (2009). Reward counteracts conflict adaptation: Evidence for a role of affect in executive control. Psychological Science, 20, 1473-1477.

van Steenbergen, H., Band, G. P. H., \& Hommel, B. (2010). In the mood for adaptation: How affect regulates conflictdriven control. Psychological Science, 21, 1629-1634.

van Steenbergen, H., Booij, L., Band, G. P. H., Hommel, B., \& Does, A. J. W. (2012). Affective regulation of cognitive-control adjustments in remitted depressive 
patients after acute tryptophan depletion. Cognitive, Affective, E Behavioral Neuroscience, 12, 280-286.

Vogt, B. A. (2005). Pain and emotion interactions in subregions of the cingulate gyrus. Nature Reviews Neuroscience, 6, 533-544.

Weinberg, A., Olvet, D. M., \& Hajcak, G. (2010). Increased error-related brain activity in generalized anxiety disorder. Biological Psychology, 85, 472-480.

Weinberg, A., Riesel, A., \& Hajcak, G. (2012). Integrating multiple perspectives on error-related brain activity: The ERN as a neural indicator of trait defensive reactivity. Motivation and Emotion, 36, 84-100.
Whitty, C., Duffield, J., \& Tow, P. (1952). Anterior cingulectomy in the treatment of mental disease. Lancet, 1, 475-481.

Yarkoni, T., Poldrack, R. A., Nichols, T. E., Van Essen, D. C., \& Wager, T. D. (2011). Large-scale automated synthesis of human functional neuroimaging data. Nature Methods, 8 , 665-670.

Yeung, N., Botvinick, M., \& Cohen, J. (2004). The neural basis of error detection: Conflict monitoring and the error-related negativity. Psychological Review, 111, 931-959.

Zaki, J., Weber, J., Bolger, N., \& Ochsner, K. (2009). The neural bases of empathic accuracy. Proceedings of the National Academy of Sciences, 106, 11382-11387. 